\title{
Analysis on the Efficacy and Safety of the Patients with Advanced Ovarian Cancer
}

\author{
Yunxia Xue ${ }^{1, a}$, Xingyun Sun ${ }^{2, *}$, Peijing Shi ${ }^{3}$ \\ ${ }^{1}$ Department of Gynecological, Third People's Hospital of Jinan, Jinan, 250132, China \\ ${ }^{2}$ Department Obstetrics and gynecology clinic, Third People's Hospital of Jinan, Jinan, 250132, \\ China \\ ${ }^{3}$ Department of Gynecological, Third People's Hospital of Jinan, Jinan, 250132, China \\ axue666@126.com
}

*Corresponding author sunxingyun@126.com

\section{Keywords: Advanced ovarian cancer; Neoadjuvant chemotherapy; Security; Effectiveness}

\begin{abstract}
Objective: To observe and explore the new adjuvant chemotherapy in patients with advanced ovarian cancer with tumour status, surgical effect and prognosis, the influence of provide a reference for clinical rational design of advanced ovarian cancer treatments. Methods: We select 32 cases in treatment group from January 2013 to December 2014, the advanced ovarian cancer treated with neoadjuvant chemotherapy. We select 32 cases of the first line of tumour to destroy loss during this period, all patients with postoperative chemotherapy, observation and comparison, the operation difficulty, tumour residual tumour foci, the differences between the recent prognosis, etc. Results: The III phase c and IV ovarian cancer neoadjuvant chemotherapy group of patients clinical effectiveness were $86.67 \%$ and $82.35 \%$ respectively; Neoadjuvant chemotherapy group patients had no chemotherapy group of patients with tumour size, water, abdominal operation difficulty, deputy damage and residual tumour foci were significantly reduced; Neoadjuvant chemotherapy group 1 year, 3 year survival rate and survival rate of patients with a median survival time were slightly higher than that of without chemotherapy group, but the differences were not statistically significant. Conclusion: The neoadjuvant chemotherapy can significantly reduce the tumour volume, reduce ascites and metastases, conducive to the operation safety and thoroughness, in spite of the recent advance and been not seen behind the obvious improvement, but still in patients with advanced ovarian cancer is one of the important treatment strategies.
\end{abstract}

\section{Introduction}

The ovarian cancer is one of the most common tumours of the female reproductive system, in the female reproductive system tumour incidence of third place, and the mortality is first in all kinds of gynaecological tumours, caused serious threat to women's health and life. Ovarian cancer early diagnosis rate is low, the diagnosis in $85 \%$ of patients had already is late, chemotherapy is the main method of treatment of advanced ovarian cancer, but is easy to produce drug resistance in the process of chemotherapy, the late pathologic staging, chemotherapy drug resistance, the easier the greater the tumour, has caused many cases of recurrence and metastasis, the 5-year survival rate of patients with only about $30 \%$. Platinum-based chemotherapy regimens of drugs is the standard treatment of ovarian cancer in the past 20 years, although its efficiency is higher, but the patient's long-term survival rate is still low, most of the patients died of tumour drug resistance. And the treatment of patients with platinum resistance is very difficult, even in the most effective drugs such as doxorubicin and cyclophosphamide treatment efficient and less than $20 \%$. To explore the effective chemotherapy of advanced ovarian cancer, this study adopts the neoadjuvant chemotherapy treatment of advanced ovarian cancer cases and compares its curative effect and adverse reactions, is presently as follows the results reported. 


\section{Materials and Methods}

\section{Materials}

We select from January 2013 to December 2014, 32 cases in treatment group, the advanced ovarian cancer treated with neoadjuvant chemotherapy. Age 37-70, the average age of 46.2 years, selection of 32 cases of the same period the first line of tumor to destroy the loss of the control group, all cases were confirmed by pathological examination, and were not surgery or a shift in after palliative resection of advanced ovarian cancer cases. Two groups of patients with no significant differences in age, illness, $\mathrm{P}>0.05$, comparable.

\section{Methods}

Treatment group receiving neoadjuvant chemotherapy, in chemotherapy of day 1, day 8 and 15 days intravenous drip polyene paclitaxel $35 \mathrm{mg} \cdot \mathrm{m}-2,1-3$ days intravenous drip in the chemotherapy of cisplatin, $25 \mathrm{mg}, \mathrm{m}-2,30$ days for a course, per patient treatment are in more than two period of treatment. 2 days before the use of paclitaxel, $8 \mathrm{mg}$ daily oral dexamethasone, taking two, even served three days, in order to reduce allergy and fluid reservoir sneak, protecting liver, anti-nausea treatment at the same time give patients, protecting stomach and other support treatment. The control group routine surgical methods, the use of all patients with postoperative chemotherapy, observation and comparison, the operation difficulty, tumor residual tumor foci, the differences between the recent prognosis, etc.

\section{Results}

III phase c and IV ovarian cancer neoadjuvant chemotherapy group of patients with clinical effectiveness were $86.67 \%$ and $82.35 \%$ respectively; Neoadjuvant chemotherapy group patients had no chemotherapy group of patients with tumor size, water, abdominal operation difficulty, deputy damage and residual tumor foci were significantly reduced; Neoadjuvant chemotherapy group 1 year, 3 year survival rate and survival rate of patients with a median survival time were slightly higher than that of without chemotherapy group, but the differences were not statistically significant. See Table 1 and Table 2

Table 1: The clinical efficacy evaluation of the ovarian cancer after chemotherapy patients

\begin{tabular}{cccccccc}
\hline Stages & CR & PR & IP & SD & PD & Total & Efficient \\
\hline IIIc Stage & 0 & 6 & 7 & 1 & 1 & 15 & 86.67 \\
IV Stage & 0 & 5 & 9 & 2 & 1 & 17 & 82.35 \\
\hline
\end{tabular}

Table 2: The intraoperative parameters between two groups of patients

\begin{tabular}{ccccccc}
\hline & $\begin{array}{c}\text { The } \\
\text { average } \\
\text { abdominal } \\
\text { water }(\mathrm{ml})\end{array}$ & $\begin{array}{c}\text { Average } \\
\text { total } \\
\text { tumor } \\
\text { diameter } \\
(\mathrm{cm})\end{array}$ & $\begin{array}{c}\text { The } \\
\text { average } \\
\text { number of } \\
\text { metastases } \\
(\mathrm{n})\end{array}$ & $\begin{array}{c}\text { The } \\
\text { average } \\
\text { operation } \\
\text { time }(\mathrm{h})\end{array}$ & $\begin{array}{c}\text { The } \\
\text { bleeding } \\
\text { in the } \\
\text { surgery } \\
(\mathrm{ml})\end{array}$ & $\begin{array}{c}\text { The optimal } \\
\text { cutting out } \\
\text { number of }\end{array}$ \\
$\begin{array}{cccccc}\text { The treatment } \\
\text { group } \\
\begin{array}{c}\text { The control } \\
\text { group }\end{array}\end{array}$ & 246 & 7.56 & 4.7 & 2.23 & 476 & 24 \\
\hline
\end{tabular}




\section{Discussion}

Neoadjuvant chemotherapy (NACT) refers to think through preoperative evaluation cannot tolerate surgery or the estimate cannot achieve ideal cut out, the first chemotherapy, and then implement cells to destroy the loss, also known as the early chemotherapy. NACT ovarian cancer include two forms: (1) by confirmed by histology ovarian cancer after several courses of chemotherapy, and row spacing (IDS) to tumor loss, the chemotherapy is called induction chemotherapy has line of tumor cells to destroy the loss, but it did not reach satisfactory tumor cells standards to cut loss, postoperative adjuvant chemotherapy for reoperation after treatment.

\section{The effect of neoadjuvant chemotherapy}

Ovarian cancer is a serious threat to women's life is one of the gynecological tumors, the cancer survival environment is good, so its early cancer symptom is not obvious, the development tend to be hidden, and sometimes menstrual changes are often ignored by patients. $70 \% \sim 70 \%$ of the patients in clinic for the first time, The out namely have been late. Ovarian malignant tumor easy spread and grows quickly. But in the early symptoms of patients are often asymptomatic or lighter, tend to stay beyond the pelvic tumor growth to a certain size, or accidentally discovered during inspection of department of gynecology, even can touch in the belly in time, or a significant complications were found, while waiting to go to a doctor often have been late. Advanced ovarian cancer neoadjuvant therapy can effectively improve the treatment effectiveness and safety, bringing light to prognosis of ovarian cancer.

Preoperative chemotherapy, neoadjuvant chemotherapy mainly through reducing tumor and surrounding tissue adhesion area, local tumor volume, reduce the degree of tumor stage, to improve the success rate of surgery, reduce complications and reduce the operative mortality. Neoadjuvant chemotherapy not only make the tumor during surgery is easy to be removed, more make many unrespectable become respectable; significantly improve the effect of the local treatment of tumor. And, through systemic chemotherapy, existing in the whole sub clinical metastases to control, so that the patient's survival rate has improved; Mass, can make tumors after neoadjuvant chemotherapy significantly narrowed, and reduce the clinical stage, make more patients get surgery opportunity; the ovarian cancer is easy to the line spread. In the first diagnosis of the patients, often more than half already exist in the whole micro metastasis. Theoretically, for there are no clinical signs and symptoms of micro metastases, to the best of the early start treatment actively, curbing its development, it has an important clinical value for improving the long-term curative effect. Malignant tumor, angiogenesis inhibiting factor can produce, which to a certain extent, can inhibit tumor development. Recent research shows that: after malignant tumor resection, angiogenesis inhibiting factor for its generation decreases, which can prompt other formation of metastases. Neoadjuvant chemotherapy can effectively prevent because of angiogenesis inhibiting factor decrease, and accelerate the tumor increased, development and metastasis.

Advanced ovarian epithelial carcinoma operation, satisfied with the first time the residual tumor diameter $<2 \mathrm{~cm}$ accounts for only about $30 \%$. The postoperative residual tumor diameter is the key factors influencing the prognosis. Neoadjuvant chemotherapy (NACT) in the treatment of advanced ovarian cancer has more than 10 years of history, is a new therapy strategy of advanced ovarian cancer. Associated with the traditional tumor cells to destroy the loss adjuvant chemotherapy, neoadjuvant chemotherapy can improve the satisfaction tumor surgery to cut loss, narrowing the scope of resection, to improve the postoperative quality of life. Neoadjuvant chemotherapy is mainly through the preoperative chemotherapy, reduce tumor and surrounding tissue adhesion and shrink the tumor volume, reduce tumor stage, improve the success rate of surgery, reduce complications and mortality. Control chest water, shorten the operation time, less intraoperative blood loss, make tumor cells into "hibernation", reduce operation caused by extrusion, such as mechanical reason tumor proliferation, planting, reduce the recurrence. New adjuvant chemotherapy for stage later, estimate the first surgery can't or can't achieve satisfactory tumor patients to cut loss, not only can improve the satisfaction ratio of tumor, at the same time can be 
within a certain range, narrowing the scope of operation, significantly reduce the blood loss, improve the quality of operation, is conducive to physical recovery after the surgery, chemotherapy.

\section{The regimens and side effects ot the chemotherapy}

Neoadjuvant chemotherapy can be divided into vein chemotherapy and intraperitoneal chemotherapy and arterial chemotherapy. Although neoadjuvant chemotherapy tumor ischemia, necrosis, tumors had narrowed, but its should not be too much, time should not be too long, the treatment is generally not more than 3 period of treatment, in order to avoid the adverse reaction of chemotherapy is not ease the timing delay surgery. Arterial chemotherapy patients taken, including 7 cases of intraperitoneal chemotherapy, the effect of 7 cases of patients after chemotherapy, disappeared basically, postoperative residual lesions $<2 \mathrm{~cm}$; Pure arterial chemotherapy in 10 cases, 6 cases of postoperative residual lesions $<2 \mathrm{~cm}$. At present many scholars believe that is the first choice for ovarian cancer neoadjuvant chemotherapy with intraperitoneal chemotherapy, and we believe that the effect of arterial chemotherapy may be better, because the arterial chemotherapy can make chemotherapy drugs in local high concentration, tumor was reduced, the benefit for the stripping of the tumor, tumor blood supply to reduce at the same time, less intraoperative bleeding. Especially a large amount of ascites in patients with advanced, arterial chemotherapy combined intraperitoneal chemotherapy can control ascites in a short time, less tumor, surgery as soon as possible, improve the satisfaction ratio of tumor surgery. But due to the number of cases is less, still need to do further research.

\section{The safety and efficacy evaluation of the treatment}

In this study, the author mainly discusses the influence of induction chemotherapy in patients with ovarian cancer surgery and prognosis. In theory, the advantage of neoadjuvant chemotherapy are the following: (1) preoperative chemotherapy can kill cancer around the foci of metastases, reduce the adhesion of tumor and the surrounding tissue, shrink the tumor volume, reduce tumor stage, improve the success rate of surgery, reduce complications and mortality; (2) control chest water, improving patient general condition, improve the tolerance of surgery; (3) to shorten the operation time, reduce intraoperative blood loss, shorten the average such confinement, improve the patient's quality of life; (4) the tumor cells into "hibernation", reduce the operation due to reasons such as extrusion, mechanical stimulation caused by tumor proliferation, planting, reduce postoperative recurrence; (5) to avoid postoperative blood coagulation mechanism to strengthen and immune suppression and easy to transfer; Those from the resection of the tumor specimen understand the sensitivity of chemotherapy. Confusion for gynecologic oncology clinicians is, however, despite more than a lot of advantage, foreign statistical data shows that the development of new adjuvant chemotherapy did not significantly improve the 5-year survival rate of patients with the ovarian cancer and the median survival time.

Based on the current domestic and relevant treatment of large sample of surgery and follow-up data, the author is from the difficulty of the tumor before and after chemotherapy, surgery and the amount of residual lesions, long-term prognosis and comprehensive analysis of the actual clinical application value of new adjuvant chemotherapy. Can be seen from this study, whether through preoperative imaging examination or intraoperative can see, suggests a new adjuvant chemotherapy can obviously reduce the patient's primary tumor volume, reduce the number of metastasis foci, so that the average total tumor focal diameter decreases obviously, and can obviously reduce the patient's abdomen. This means that the role of neoadjuvant chemotherapy on tumor tissue is clearly and definitely, and can increase the tolerance of surgery patients; the conclusion is consistent with results. This study also found that line preoperative neoadjuvant chemotherapy can not only reduce tumor foci, and reduces the difficulty of operation and reduce the complications. Neoadjuvant chemotherapy group of patients with preoperative blood loss and operation time were significantly less than in patients with no chemotherapy group, and no one vice injury surgery and its difference shows that preoperative neoadjuvant chemotherapy can improve the success rate of surgery, the treatment of advanced ovarian cancer has important value. In this study, although the new adjuvant chemotherapy group of patients with no chemotherapy group 1 year, 3 year survival rate and 
survival rate of patients with a median survival time were no significant difference, but all training to learn one data still showed a more than patients with neoadjuvant chemotherapy group index higher than no chemotherapy group, prompt the long-term prognosis is slightly better than no patients with chemotherapy group. Domestic scholars in 51 patients underwent neoadjuvant chemotherapy for follow-up after had a similar conclusion. For neoadjuvant chemotherapy did not significantly improve the prognosis of patients with ovarian cancer, had a foreign scholars think that preoperative chemotherapy can induce tumor cells to produce drug resistance and bring adverse effect to the postoperative chemotherapy, but so far there is no direct evidence, the argument is worth further research.

Think at present, by preoperative neoadjuvant chemotherapy can reduce the narrow scope of intra-abdominal lesions, tumor load, reduce the complications, shorten the total length of hospital stay and time in the ICU. Most were retrospectively analyzed according to neoadjuvant chemotherapy treatment does not affect survival. Although it is not clear whether the new adjuvant chemotherapy can improve survival, but for the first time procedures cannot achieve satisfactory reduction of tumor surgery or surgery in patients with advanced ovarian cancer, is still an important treatment strategy. Therefore, it is necessary to further study of neoadjuvant chemotherapy in patients with ovarian cancer survival time, complications, treatment and related mortality, and survival quality, efficiency, etc, the influence of clear its role in the treatment of ovarian cancer for the first time and meaning.

\section{Conclusion}

The analysis shows that the advanced ovarian cancer preoperative neoadjuvant chemotherapy 13 courses can better control the chest, ascites, effectively improve the whole body, improve the body's tolerance to surgery patients, and can eliminate the liver, lungs and other distant metastases, reduce tumor stage, increase operation feasibility, can shrink the tumor volume at the same time, reduce or loose the adhesion of tumor and normal tissue, effectively reduce the harmful surgery, kill celiac tumor metastases in the short term, to shorten the operation time, reduce intraoperative bleeding and improve the success rate of tumor cells to destroy the loss of purpose. Patients can tolerate the side reaction of neoadjuvant chemotherapy. This study proves that the neoadjuvant chemotherapy can significantly reduce the tumor volume, reduce ascites and metastases, conducive to the operation safety and thoroughness, in spite of the recent advance and been not seen behind the obvious improvement, but still in patients with advanced ovarian cancer is one of the important treatment strategies.

\section{Acknowledgement}

This research was financially supported by the National Science Foundation.

\section{References}

[1] Ping Guo, Jianguo Ma, Shaolan Li, et al. Determination of paclitaxel in mouse plasma and brain tissue by liquid chromatography's spectrometry. Journal of Chromatography B: Analytical Technologies in the Biomedical and Life Sciences. 2013

[2] Cunnistra S A. Cancer of the ovary. New England Journal of Homeopathy. 2012

[3] Raymond E, Lawrence R, Zbicka E, et al. Activity of oxali-platin against human tumor colony-forming units. Clinical Cancer Research. 2013

[4] Hannigan,E, Green,S, Alberts,D, O, TR, Surwit, E. , Results of a Southwest Oncology Group Phase III trial of carboplatin plus cyclophosphamide versus cisplatin plus cyclophosphamide in advanced ovarian cancer. Oncology. 2013

[5] Dercksen MW, Hoekman K, ten Bokkel Huinink WW, et al. Effects of interleukin-3 on myelo-suppression induced by chemotherapy for ovari-an cancer and small cell undifferentiated tumours. British Journal of Cancer. 2011 
[6] R.A. Burger, P.J. Di Saia, J.A. Roberts. Phase II trial of vinorelbine in recurrent and progressive epithelial ovarian cancer. Gynecologic Oncology. 1999

[7] Bristow RE, Palis BE, Chi DS, et al. The National Cancer Database report on advanced-stage epithelial ovarian cancer: impact of hospital surgical case volume on overall survival and surgical treatment paradigm. Gynecologic Oncology. 2012

[8] Minagawa Y, Kigawa J, Kanamori Y, et al. Feasibility study comparing docetaxel-cisplatin versus docetaxel-carboplatin as first-line chemotherapy for ovarian cancer. Gynecologic Oncology. 2012

[9] Lee.C.B. Therapeutic advances in local-regional therapy for stage III non-small-cell lung cancer: evolving role of dose-escalated conformal (3-dimensional)radiation therapy. Clinical Lung Cancer Journal. 2012

[10] Martel M K,Ten Haten R K,Hazuka M B, et al. Estimation of tumor control probability model parameters from 3-D dose distributions of non-small cell lung cancer patients. Lung Cancer. 2010 